\title{
Uso de métodos fotográficos no contexto da saúde mental: Uma revisão integrativa
}

\author{
Gabriela Trombeta, Lívia Scienza e Maria de Jesus D. dos Reis \\ Programa de Pós-Graduação em Psicologia, Universidade Federal de São Carlos (UFSCar)
}

Submissão: 25 jun. 2020.

Aceite: 18 maio 2021.

Notas das autoras

Gabriela Trombeta (D) https://orcid.org/00oo-0001-5485-1239

Lívia Scienza (D) https://orcid.org/00oo-0003-1797-1949

Maria de Jesus D. dos Reis (D) https://orcid.org/000o-0002-5128-2074

Esta pesquisa foi financiada pela Coordenação de Aperfeiçoamento de Pessoal de Nível Superior (Capes) - Código de Financiamento 001 - e Fundação de Amparo à Pesquisa do Estado de São Paulo (Fapesp) - Processo no 2018 / 10632-8.

Correspondências referentes a este artigo devem ser enviadas para Gabriela Trombeta, Via Washington Luis, Km 235, São Carlos, SP, Brasil. CEP 13565-905. E-mail: gabriela_trombeta@ hotmail.com 


\title{
Resumo
}

Métodos que utilizam da produção de fotografias têm sido explorados como instrumentos alternativos de pesquisa qualitativa e intervenção psicológica. Entretanto, ainda há pouco consenso em suas denominações e procedimentos. Nesse contexto, a presente revisão integrativa visa investigar o uso de métodos fotográficos no campo da saúde mental, nos últimos 20 anos. A pesquisa foi realizada nas bases de dados LILACS, PsycNET, PubMed, SciELO e Web of Science. Incluíram-se 49 artigos, e excluíram-se 457. Identificaram-se os métodos: fotovoz, instrumento fotográfico, autofotografia e fotoelicitação. Como potencialidades, destacou-se o uso da fotografia como meio para explorar e compartilhar experiências internas, auxiliar profissionais de saúde e gerar empoderamento. Como desafios, prevaleceram aspectos relacionados ao caráter recente do uso de métodos fotográficos no campo da saúde mental, como: predomínio de amostras pequenas e não generalizáveis, múltiplas metodologias de análise de dados e utilizações inadequadas de terminologias em relação aos procedimentos realizados.

Palavras-chave: fotografia, psicologia, pesquisa, métodos visuais, revisão

\section{USING PHOTOGRAPHIC METHODS IN THE MENTAL HEALTH FIELD: AN INTEGRATIVE REVIEW}

\begin{abstract}
Methods using production of photographs have been explored as alternative instruments of qualitative research and psychological intervention. However, there is still little consensus in their designations and procedures. In this context, this work presents an integrative review aimed at investigating the use of photographic methods in the field of mental health over the last 20 years. The research was conducted on LILACS, PsycNET, PubMed, SciELO and Web of Science databases. Forty-nine articles were accepted and 457 rejected. Four methods were identified: photovoice, photo-instrument, autophotography and photo-elicitation. As potential aspects, the use of photography means exploring and sharing personal experiences, assisting health professionals and creating empowerment. As challenge aspects, the recent feature of photographic methods used in the field of mental health were highlighted, counting on the prevalence of small and non-generable samples, multiple data analysis methodologies and inappropriate uses of designations regarding the procedures that were performed.
\end{abstract}

Keywords: photography, psychology, research, visual methods, review

\section{USO DE MÉTODOS FOTOGRÁFICOS EN EL ÁMBITO DE LA SALUD MENTAL: UNA REVISIÓN INTEGRATIVA}

\section{Resumen}

Métodos que utilizan la producción de fotos han sido explorados como instrumentos alternativos de investigación cualitativa y intervención psicológica. Sin embargo, aún hay poco consenso en relación a sus denominaciones y procedimientos. Esta revisión integrativa investiga el uso de métodos fotográficos en el campo de la salud mental en los últimos 20 años. La investigación fue realizada en las bases 
LILACS, PsycNET, PubMed, SciELO y Web of Science. Cuarenta y nueve artículos fueron aceptos y 457 excluidos. Se identificaron los siguientes métodos: fotovoz, instrumento fotográfico, autofotografía y fotoelicitación. Como potencialidad ese destacó el uso de fotografías como un medio para explorar y compartir experiencias internas, ayudar a los profesionales de la salud y generar empoderamiento. En los desafíos prevalecieron la caracterización aún reciente del uso de métodos fotográficos en la salud mental, el predominio de muestras pequeñas y no generalizables, múltiples metodologías de análisis de datos y inadecuado de terminologías.

Palabras clave: fotografía, psicología, investigación, métodos visuales, revisión 
Diversos métodos visuais têm sido utilizados em pesquisas qualitativas como alternativas a métodos verbais e escritos. O uso de imagens e objetos durante uma pesquisa parece auxiliar os participantes na evocação de pensamentos mais profundos e críticos sobre determinado assunto, facilitar a articulação e verbalização de suas experiências, e contribuir para seu engajamento e empoderamento, de modo a colocá-los como sujeitos ativos e fundamentais para a produção do conhecimento e, ao mesmo tempo, colaborar para o enriquecimento dos dados obtidos pelos pesquisadores (Glaw et al., 2017; Padgett et al., 2013; Piat et al., 2017).

Apesar de este artigo se referir apenas ao uso de fotografias, métodos visuais podem incluir diversos materiais visuais, como pinturas, esculturas, desenhos, vídeos, fotografias, poemas, artefatos e objetos. Tais materiais podem caracterizar-se como instrumentos facilitadores do diálogo, como dados em si ou como ambos simultaneamente (Sitvast et al., 2010; Padgett et al., 2013).

No caso de serem utilizados apenas como instrumentos, pode-se considerar o material visual como um suporte para entrevistas ou grupos focais, como uma maneira de facilitar a comunicação de temáticas, assuntos ou ideias específicos. É possível, por exemplo, mostrar a participantes fotos sobre determinado assunto e fazer perguntas sobre o tema a ser investigado a partir do conteúdo das fotografias. Nessas circunstâncias, as fotos são apenas um suporte no qual pesquisadores e participantes se apoiam para desenvolver a conversa, não sendo necessariamente produzidas pelos participantes do estudo. Todavia, além do seu uso como um instrumento facilitador, as fotos podem também ser utilizadas como dados em pesquisas qualitativas, ao solicitarmos aos participantes que tirem fotos sobre determinado assunto e depois compartilhem com os pesquisadores. Nesse cenário, as fotografias ocupam o papel não apenas de apoio para o diálogo, mas também de dados de pesquisa, uma vez que as fotos podem conter informações relevantes sobre a visão daquela pessoa a respeito do assunto investigado.

O uso de fotografias tiradas por participantes é recorrente em pesquisas de diferentes áreas do conhecimento, como em estudos de antropologia (Collier, 1957), ciências sociais (Smith et al., 2015; Vélez-Grau, 2018), educação (Vilà et al., 2016), terapia ocupacional (Maniam et al., 2016; Greco et al., 2016), enfermagem (Clements, 2012), psiquiatria (Russinova et al., 2018; Sibeoni et al., 2017) e psicologia (Oliffe et al., 2017; Quaglietti, 2018). No entanto, apesar de esse tipo de metodologia (a qual chamaremos aqui de métodos fotográficos) estar presente em diferentes áreas do conhecimento, diversos pesquisadores têm mencionado seu papel inovador e seus benefícios principalmente em pesquisas no contexto da saúde mental (Glaw et al. 2017; Erdner et al., 2009). Isso acontece por ser comum que essas metodologias envolvam temas difíceis de falar, experiências emocionais intensas e populações com pouco repertório em verbalizar vivências de forma mais expressiva ou direta (Creighton et al., 2013; Drew et al., 2010; Padgett et al., 2013). 
Nesse contexto, o uso de métodos fotográficos tem se demonstrado eficaz em valorizar e capturar com mais detalhes as experiências emocionais vividas pelos que estão sendo ouvidos (Erdner et al., 2009), o que é relevante para desconstrução de estigmas relacionados à saúde mental e para auxiliar profissionais e acadêmicos a compreender mais profundamente as demandas da população com que estão trabalhando. Tal aspecto parece ser possível pelo fato de que as fotografias contribuem para a aproximação de pesquisadores e participantes (Tittoni, 2009) e também para o acesso a dados que podem complementar as informações obtidas apenas por meio de questionários e entrevistas tradicionais (Drew \& Guillemin, 2014; Glaw et al., 2017; Padgett et al., 2013). Sendo assim, são especialmente úteis no diálogo com populações que vivenciam transtornos mentais e apresentam dificuldade em expressar seus pensamentos e sentimentos apenas por meios verbais e/ou em situações sociais vistas como intimidadoras.

Ainda que os potenciais do uso de métodos que utilizam fotografias tiradas por participantes sejam reconhecidos pela literatura em saúde mental, Glaw et al. (2017) indicam que tais métodos têm sido subutilizados nesse contexto. Um dos fatores pelos quais isso acontece parece ser a grande diversidade de denominações e procedimentos utilizados para se referir a esses métodos, dificultando que eles se tornem acessíveis e compreensíveis a pesquisadores e profissionais que desejam começar a utilizá-los no campo da saúde mental.

Considerando esses aspectos e a escassez de literatura sistematizando o uso de métodos fotográficos no contexto da saúde mental, esta pesquisa tem como objetivo realizar uma revisão integrativa sobre esse tema, buscando responder às seguintes perguntas: Que métodos fotográficos têm sido utilizados no contexto da saúde mental? Quais são as metodologias envolvidas em suas aplicações? Como os dados produzidos por eles têm sido analisados? Quais efeitos de seu uso têm sido apontados pela literatura?

\section{Método}

Foram consultadas as bases de dados LILACS, PsycNET, PubMed, SciELO e Web of Science, considerando as strings apresentadas na Tabela 1. Tais strings foram escolhidas para esta revisão após uma busca pela combinação de palavras "Fotografia AND Saúde Mental/ Photography AND Mental Health/Fotografia AND Salud Mental", realizada nessas bases de dados. Isso foi feito com o intuito de identificar quais termos eram utilizados na literatura para se referir ao uso de fotografias tiradas por participantes em pesquisas no contexto da saúde mental. Logo, as strings escolhidas para este estudo (Tabela 1) se referem aos métodos fotográficos encontrados nesta pesquisa prévia. 


\section{Tabela 1}

Strings utilizadas para a busca dos artigos nas bases de dados

\begin{tabular}{|c|c|c|}
\hline Inglês & Português & Espanhol \\
\hline autophotography OR & autofotografia OR & autofotografia OR \\
\hline auto-photography & autofotografia & auto-fotografia \\
\hline AND mental health & AND saúde mental & AND salud mental; \\
\hline photovoice AND mental health & fotovoz AND saúde mental; & photovoice AND salud mental \\
\hline $\begin{array}{l}\text { hermeneutic photography AND } \\
\text { mental health }\end{array}$ & $\begin{array}{l}\text { fotografia hermenêutica AND } \\
\text { saúde mental }\end{array}$ & $\begin{array}{l}\text { fotografia hermeneutica AND } \\
\text { salud mental }\end{array}$ \\
\hline $\begin{array}{l}\text { photocomposition AND mental } \\
\text { health }\end{array}$ & $\begin{array}{l}\text { fotocomposição AND } \\
\text { saúde mental }\end{array}$ & $\begin{array}{l}\text { fotocomposición AND } \\
\text { salud mental }\end{array}$ \\
\hline $\begin{array}{l}\text { photo interview AND mental } \\
\text { health }\end{array}$ & $\begin{array}{l}\text { foto entrevista OR entrevista } \\
\text { fotográfica AND saúde mental }\end{array}$ & $\begin{array}{l}\text { foto entrevista OR entrevista } \\
\text { fotográfica AND salud mental }\end{array}$ \\
\hline $\begin{array}{l}\text { photo stories AND mental } \\
\text { health }\end{array}$ & fotobiografia AND saúde mental & fotobiografia AND salud mental \\
\hline $\begin{array}{l}\text { photo-elicitation AND mental } \\
\text { health }\end{array}$ & $\begin{array}{l}\text { fotoelicitação AND } \\
\text { saúde mental }\end{array}$ & $\begin{array}{l}\text { foto-provocación OR } \\
\text { foto-elucidación AND } \\
\text { salud mental }\end{array}$ \\
\hline
\end{tabular}

A pesquisa e a seleção dos artigos foram realizadas no período de outubro de 2018 a janeiro de 2019. Utilizaram-se os seguintes critérios de inclusão: 1. artigos em inglês, português ou espanhol; 2. publicados em revistas revisadas por pares; 3. publicados no período de 1999 a 2019; 4. estudos empíricos referentes à saúde mental; 5. fotografias tiradas pelos participantes do estudo; 6 . estudos com participantes com indicadores de transtornos mentais (com diagnósticos e/ou em tratamento clínico e/ou fazendo uso de medicações relacionadas à saúde mental). Os critérios de rejeição foram: 1. artigos em outras línguas; 2. publicados em revistas não revisadas por pares; 3. publicados antes de 1998; 4. estudos não empíricos e/ou não relacionados à saúde mental; 5 . fotografias não tiradas pelos participantes do estudo; 6 . com participantes sem indicativo de transtornos mentais. Rejeitaram-se todos os artigos duplicados e que não atendiam a qualquer um dos critérios de inclusão. Para que os artigos fossem aceitos, eles deveriam atender a todos os critérios de inclusão mencionados. Exemplos de títulos de artigos que atendiam aos critérios de inclusão 4, 5 e 6 encontram-se disponíveis na Tabela 2. Os demais critérios de inclusão (1, 2 e 3) não foram contemplados por concentrarem-se em restrições de idioma, data de publicação e revisão por pares. 
Tabela 2

Exemplos de títulos de artigos enquadrados nos critérios de inclusão 4, 5 e 6

\begin{tabular}{|c|c|}
\hline Critérios de inclusão & Exemplos de títulos \\
\hline \multirow[t]{6}{*}{$\begin{array}{l}\text { Estudos empíricos } \\
\text { referentes à saúde mental }\end{array}$} & $\begin{array}{l}\text { "Understanding the experiences of survivors of a loss by suicide: A } \\
\text { photovoice study" }\end{array}$ \\
\hline & $\begin{array}{l}\text { "Use of photovoice to understand the experience of taking psychotropic } \\
\text { medications" }\end{array}$ \\
\hline & $\begin{array}{l}\text { "Correlates of autophotographic individuality: Therapy experience and } \\
\text { loneliness" }\end{array}$ \\
\hline & $\begin{array}{l}\text { "Using photovoice as a method to engage bereaved adults with } \\
\text { intellectual disabilities in research: Listening, learning and developing } \\
\text { good practice principles" }\end{array}$ \\
\hline & $\begin{array}{l}\text { "Experiencing recovery: Findings from a qualitative study into mental } \\
\text { illness, self and place" }\end{array}$ \\
\hline & $\begin{array}{l}\text { "Imagining inclusion uncovering the upstream determinants of mental } \\
\text { health through photovoice" }\end{array}$ \\
\hline \multirow[t]{6}{*}{$\begin{array}{l}\text { Fotografias tiradas pelos } \\
\text { participantes do estudo }\end{array}$} & $\begin{array}{l}\text { "Reframing autism: Young adults with autism share their strengths } \\
\text { through photo-stories" }\end{array}$ \\
\hline & "Facades of suffering: Clients' photo stories about mental illness" \\
\hline & $\begin{array}{l}\text { "New roads paved on losses: Photovoice perspectives about recovery from } \\
\text { mental illness" }\end{array}$ \\
\hline & $\begin{array}{l}\text { "Using photovoice to examine adolescents' experiences receiving mental } \\
\text { health services in the United States" }\end{array}$ \\
\hline & $\begin{array}{l}\text { "Using photovoice to explore patients' experiences with mental health } \\
\text { medication: A pilot study" }\end{array}$ \\
\hline & $\begin{array}{l}\text { "The big picture unfolds: Using photovoice to study user participation in } \\
\text { mental health services" }\end{array}$ \\
\hline \multirow{4}{*}{$\begin{array}{l}\text { Estudos com participantes } \\
\text { que possuíam indicadores } \\
\text { de transtornos mentais }\end{array}$} & $\begin{array}{l}\text { "(Em)placing recovery: Sites of health and wellness for individuals with } \\
\text { serious mental illness in supported housing" }\end{array}$ \\
\hline & $\begin{array}{l}\text { "Photo-elicitation with adolescents in qualitative research: An example of } \\
\text { its use in exploring family interactions in adolescent psychiatry" }\end{array}$ \\
\hline & $\begin{array}{l}\text { "Quality of life for young adults with intellectual disability following } \\
\text { individualized support: Individual and family responses" }\end{array}$ \\
\hline & $\begin{array}{l}\text { "The subjective experience and phenomenology of depression following } \\
\text { first episode psychosis: A qualitative study using photo-elicitation" }\end{array}$ \\
\hline
\end{tabular}

Para reduzir vieses na seleção dos artigos a serem analisados, esse processo foi realizado, inicialmente, por duas pesquisadoras. Cada uma pesquisou as strings nas bases de dados indicadas e baixou seus respectivos resultados no software Parsif.al (https://parsif.al/). Em seguida, ambas tiveram acesso aos critérios de inclusão e exclusão do estudo e fizeram uma seleção dos artigos por meio da leitura do resumo dos estudos encontrados. Caso o resumo não contemplasse informações suficientes para a inclusão ou exclusão, o artigo completo era consultado. Após a condução desse processo de forma individual, cada pesquisadora gerou 
uma tabela com os artigos aceitos e rejeitados, indicando ao menos uma justificativa. No caso de artigos com julgamentos distintos, uma terceira pesquisadora, selecionada por afinidade com o tema tratado e por sua vasta experiência na condução de revisões de literatura, foi responsável pela decisão final, tomada após leitura dos julgamentos anteriores e verificação dos textos completos dos estudos.

Concluído o processo de seleção, uma análise detalhada dos estudos aceitos foi realizada pela primeira pesquisadora. Isso foi feito também no software Parsif.al, contando com a criação de um formulário de extração com itens relevantes a serem preenchidos durante a leitura, sendo eles: autor; ano de publicação; universidade dos autores; país; periódico; objetivo do estudo; população-alvo; método fotográfico utilizado; propósito para utilização; população; instruções dadas aos participantes; número de encontros e duração; número de fotos solicitadas; tempo dado aos participantes para que pudessem tirar as fotos; entrevistas individuais ou grupos de debate; análise de dados; contribuições; limitações; e informações adicionais.

Após a leitura e o preenchimento dos formulários referentes a cada artigo, realizaram-se, manualmente, análises quantitativas para cada um dos itens supracitados. Nessa etapa, registraram-se as análises em tabelas referentes aos itens a que pertenciam, e agruparam-se os itens repetidos, os quais foram organizados em ordem crescente. Por fim, analisaram-se criticamente os dados obtidos, e realizaram-se uma interpretação dos resultados e a síntese de seus pontos mais relevantes.

\section{Resultados}

Encontraram-se 506 artigos, dos quais 49 foram aceitos de acordo com os critérios de inclusão, e foram excluídos 191 por duplicidade e 266 por não atenderem aos critérios de inclusão' (Kappa $=0,801$ ). Em relação aos artigos aceitos, suas publicações têm sido mais prevalentes no período de 2016 a 2018. O fluxograma desse processo de seleção dos artigos, assim como a distribuição dos artigos ao longo dos anos, encontra-se ilustrado na Figura 1.

A lista de artigos aceitos está disponível em: https://github.com/gtrombeta/Fotografia-e-psicologia artigo/blob/ master/ANEXO\%201\%20-\%20Refer\%C3\%AAncias\%20bibliogr\%C3\%A1ficas\%20dos\%20artigos\%20aceitos.pdf 


\section{Figura 1}

Fluxograma das etapas da revisão sistemática e seus respectivos resultados

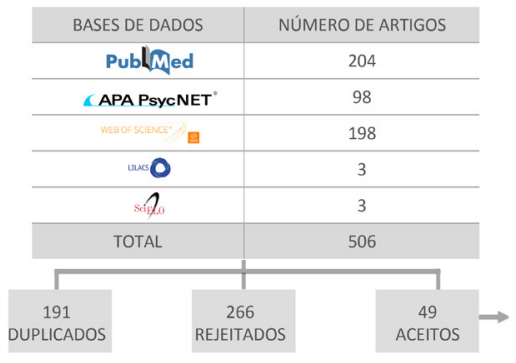

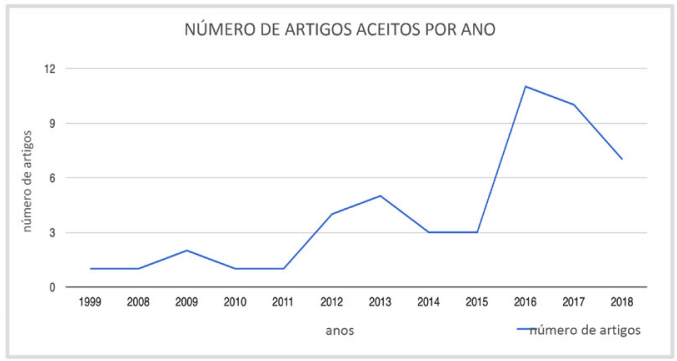

\section{Métodos fotográficos}

Foram encontradas referências de quatro maneiras de utilização de fotografia tirada por participantes no contexto da saúde mental, sendo elas: photovoice (fotovoz), photo-elicitation (fotoelicitação), photo-instrument (instrumento fotográfico) e autophotography (autofotografia). Alguns estudos contam também com a combinação de procedimentos, somando autofotografia e fotoelicitação (Glaw et al., 2017) ou fotovoz e fotoelicitação (Oliffe, et al., 2017). A Figura 2 retrata o número de artigos se referindo a cada método.

\section{Figura 2}

Gráfico e tabela referente aos métodos fotográficos encontrados e números de artigos se referindo a cada um deles

\begin{tabular}{|c|c|}
\hline Métodos fotográficos & № Art. \\
\hline Fotovoz & $32 \quad \square$ \\
\hline Fotoelicitação & $9 \quad \square$ \\
\hline Instrumento fotográfico & $3 \quad \square$ \\
\hline Fotovoz + Fotoelicitação & $2 \quad \square$ \\
\hline Autofotografia & 2 \\
\hline Autofotografia + Fotoelicitação & 1 \\
\hline Total de artigos & 49 \\
\hline
\end{tabular}

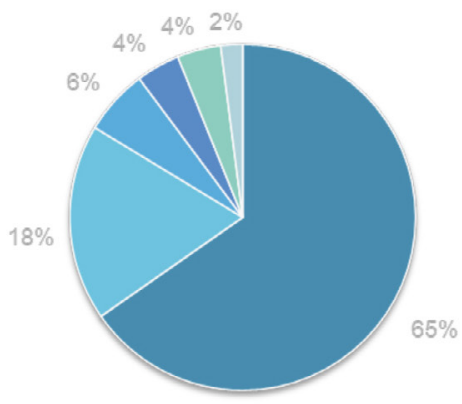

Por conta da identificação de contradições nas descrições desses métodos nos artigos analisados, suas definições e particularidades serão descritas utilizando como base as referências bibliográficas dos autores pioneiros em desenvolvê-los. 
A fotoelicitação tem suas origens na antropologia (Collier, 1957) e consiste no uso de fotografias em uma entrevista individual a fim de evocar pensamentos e falas no participante, pedindo a este que olhe as fotografias e responda a algumas perguntas. Uma vez que as fotografias se caracterizam apenas como material de apoio para o incentivo ao diálogo e uma forma de enriquecer os dados, nesse método elas não ocupam a função de dados per se. Sendo assim, podem ser tiradas pelos participantes da entrevista ou tiradas por um terceiro, escolhidas de arquivos pessoais ou escolhidas pelos pesquisadores. Quando as fotografias são tiradas pela pessoa a ser entrevistada, o procedimento geralmente acontece em dois encontros, sendo o primeiro para direcionamentos e instruções, e o segundo para que o participante discorra sobre as fotografias.

Já sobre a autofotografia, seus primeiros registros remontam a pesquisas da psicologia social (Ziller, 1990), consistindo em pedir aos participantes que tirem fotos em resposta a uma pergunta e/ou tema e as entreguem ao pesquisador que, então, analisará as fotografias com o objetivo de explorar experiências subjetivas do sujeito. Para esse método, as fotografias são os dados per si, e, portanto, as fotos precisam necessariamente ser tiradas pelo participante, sendo opcional o uso de entrevistas para que ele dê mais detalhes sobre suas fotos.

O método fotovoz, por sua vez, tem origem em pesquisas de saúde (Wang \& Burris, 1997) e é descrito como uma pesquisa-ação participativa, ${ }^{2}$ destacando-se por utilizar-se da tomada de fotografias como meio de identificação, representação e discussão de assuntos relevantes para uma comunidade específica. Com esse objetivo, o pesquisador ou profissional trabalha com os grupos, formados por membros de uma mesma comunidade, para identificar um tema sobre o qual considerem importante dialogar. Decidido o tema, é solicitado aos membros da comunidade que tirem fotos refletindo sua visão a respeito dele. Em seguida, o responsável pelo procedimento se reúne com os membros e guia grupos de discussões focais sobre as fotografias tiradas por eles, com o principal objetivo de os diálogos evocados funcionarem como um estímulo para possíveis mudanças sociais. Como há a intenção de intervenção, a fotovoz usualmente conta com durações mais prolongadas, geralmente de quatro a dez encontros, realizados uma vez por semana. É possível adicionar ao método fotovoz entrevistas individuais e também é comum a organização de uma exposição fotográfica ao final do procedimento, como maneira de compartilhar as fotos e discussões produzidas com profissionais de saúde ou com a própria comunidade (Maniam et al., 2016; Paton et al., 2018; Quaglietti, 2018; Teti et al., 2016; Werremeyer et al., 2016)

Já o instrumento-fotográfico é uma adaptação do fotovoz (Sitvast \& Abma, 2011). Tal método compartilha com o fotovoz o procedimento de solicitar fotografias aos participantes e discuti-las em grupos, em períodos mais prolongados e com intenção de intervenção. No en-

2 Tradução livre da expressão original: participatory-action research (Wang, 1999). 
tanto, diferencia-se no sentido de que os temas a serem fotografados não se relacionam a uma comunidade, mas sim a processos individuais, possuindo como principal objetivo a construção e/ou reconstrução de significados de uma experiência como um processo terapêutico. Outras expressões relacionadas ao instrumento-fotográfico citados por Sitvast e Abma (2011) são histórias fotográficas, grupos fotográficos e fotografia hermenêutica. ${ }^{3}$

\section{Procedimentos comuns}

Os quatro métodos contam com um procedimento em comum: a tarefa de solicitar aos participantes que tirem fotos em relação a determinada pergunta ou tema, dar a eles um intervalo para a tomada das fotos e em seguida pedir que tragam as fotografias ao pesquisador ou profissional, sendo possível que as imagens sejam apenas entregues ou que haja uma posterior discussão sobre elas de acordo com o método fotográfico que está sendo utilizado. No caso de haver discussão, as sessões são gravadas e transcritas para posterior análise qualitativa. Ademais, todos os métodos fotográficos também contam com a possibilidade de adicionar a seu delineamento outros instrumentos relevantes ao objetivo da pesquisa, como documentos de informações demográficas, escalas Likert, escalas validadas ou construídas pelos pesquisadores, diários de campo e escrita de narrativas.

\section{Figura 3}

Descrições das especificidades de cada método fotográfico encontrado
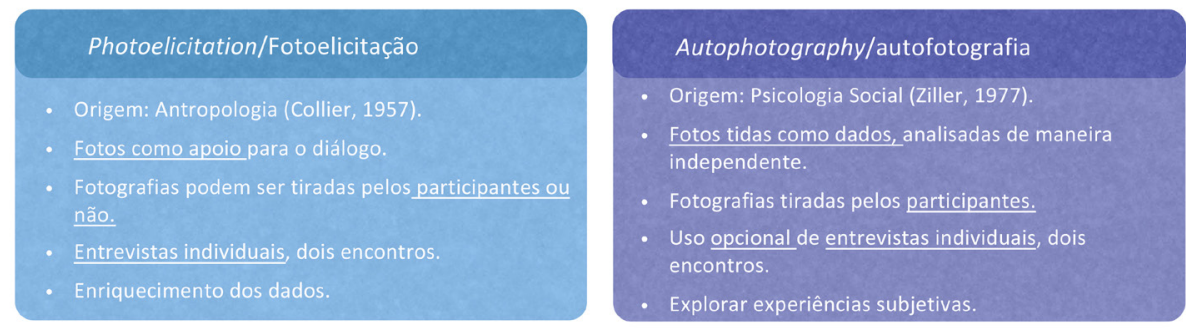

Photovoice/Fotovoz

Photo-Instrument/Instrumento Fotográfico

- Origem: Saúde (Wang \& Burris, 1997).

- Fotografias tiradas por participantes.

Temas relacionados à comunidade.

Discussões em grupo ou grupo + entrevista

individual + exposições, 4-10 encontros.

Usadas como estimulo para possiveis mudanças sociais.

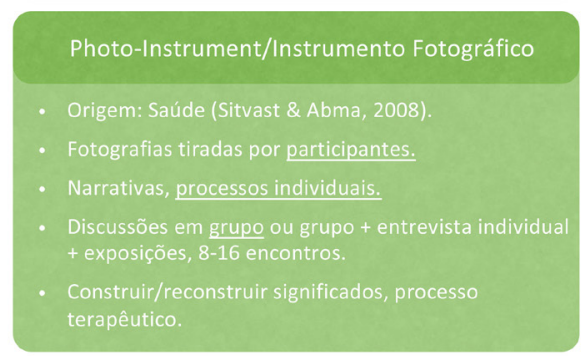

3

Tradução livre. As expressões originais em inglês são, respectivamente, photostories, photogroups e hermeneutic photography. 
Como visto nas descrições das especificidades de cada método e em sua síntese presente na Figura 3, a configuração das discussões e o número de encontros variam de acordo com o método escolhido, podendo acontecer em sessões individuais ou em grupos por meio de entrevistas semiestruturadas com perguntas abertas. Apesar de não haver uma padronização nas perguntas a serem realizadas, o uso de um conjunto de cinco perguntas nomeado Método SHOWED foi identificado em 14 dos artigos selecionados. Tal conjunto foi proposto por Wang et al. (2004) e consiste nas seguintes perguntas: "O que você vê aqui?", "O que realmente está acontecendo?", "Como isso está relacionado à nossa vida?", "Por que esse problema ou força existe?" e "O que podemos fazer sobre isso?".4

Já em relação ao intervalo de tempo dado para tomada das fotos e quantidade de fotos solicitadas, não há consenso e número determinado, variando em função das condições de pesquisa dos autores. Nos artigos analisados, o intervalo de tempo dado para a tomada das fotografias variou de uma a quatro semanas, e a quantidade de fotos solicitadas variou de uma a 27 , incluindo a possibilidade de a quantidade de fotos não ser limitada no primeiro momento, e, posteriormente, no momento do diálogo sobre elas, pedir aos participantes que escoIham quais são as fotos mais importantes para ele, de quais gostaram mais ou sobre quais gostariam de discorrer. Essa opção é dada como uma forma de permitir que os participantes façam um primeiro recorte dos dados e também a fim de tornar o diálogo menos cansativo.

A respeito do tipo de população em que os métodos fotográficos vêm sendo utilizados, identificou-se sua aplicação a amostras pequenas, destacando-se estudos relacionados a adultos com doenças mentais severas, duplo diagnóstico ou deficiência intelectual e adolescentes recebendo cuidados psiquiátricos. A identificação de tal população específica está diretamente relacionada a um dos critérios de inclusão dos artigos desta revisão: o indicativo de transtorno psicológico nos participantes, seja por meio de relatos de diagnóstico, uso de medicações psicotrópicas ou pelo fato de estarem em instituições de cuidados de saúde mental. Dessa forma, é importante ressaltar que tais métodos também são empregados em estudos com populações de diferentes faixas etárias, condições sociais e de saúde, sendo possível que a não identificação desse excerto populacional se deva apenas ao delineamento do presente estudo.

\section{Propósitos e efeitos da utilização de fotografias tiradas por participantes em pesquisas no contexto da saúde mental}

Como propósitos para aplicação de métodos fotográficos, os autores dos artigos analisados justificam, predominantemente, a utilização da fotografia como um meio para compartilhar e explorar experiências internas e auxiliar profissionais na compreensão de experiên-

$4 \quad$ Tradução livre das perguntas originais: "What do you see here?", "What is really happening?", "How does this relate to our lives?," "Why does this problem or strength exist?" e "What can we do about it?". 
cias subjetivas individuais. Foram igualmente recorrentes as referências ao uso da fotografia como apoio para entrevistas, como medida de autorrelato e como forma de intervenção por meio da facilitação do processo de engajamento, recuperação, atribuição de significados ou ressignificação de uma experiência e de motivação para mudanças sociais.

Quanto aos efeitos observados após a realização dos procedimentos, os autores dos estudos destacam que a criação de um espaço para expressão e diálogo derivada do uso das fotografias gerou empoderamento dos participantes, facilitou o surgimento de informações relevantes a pesquisadores e profissionais e também iniciou nos participantes um processo de autorreflexão crítica sobre a vida pessoal. Tal autorreflexão é usualmente constatada por meio de declarações de maior consciência de si e do processo pessoal de recuperação durante a tomada das fotografias. Alguns estudos relatam também que as fotografias ocuparam o papel de estabelecer uma ponte entre os eventos vividos e sua articulação verbal, auxiliando na ressignificação de experiências e facilitando o engajamento dos participantes na pesquisa.

\section{Análise de dados envolvendo fotografias e entrevistas}

Identificaram-se duas possibilidades predominantes em relação às quais dados obtidos por meio de métodos fotográficos são levados em consideração e em que sequência são analisados, sendo elas: 1. análise independente das transcrições das entrevistas seguida da junção de fotografias e entrevistas para análise conjunta e 2. junção das fotografias às transcrições das entrevistas de cada participante para análise conjunta.

No que diz respeito ao embasamento teórico para as análises, identificaram-se diversas metodologias e abordagens, sendo as mais utilizadas: análise temática segundo o modelo de Braun e Clarke (2006) e a teoria fundamentada de Strauss e Corbin (1998) ou Charmaz (2006). Outras formas de análise identificadas foram: análises hermenêuticas, análise semiótica e iconográfica, análises de regressão linear de efeitos mistos, análises de conteúdo e análises discursivas. Houve também referências à utilização de softwares para análise dos dados, sendo oito delas referentes ao Atlas.ti, quatro ao NVivo e uma ao Stata11.

Como meio de garantir a fidedignidade dos dados, procedimentos bastante citados foram a triangulação dos dados e a realização de análises independentes por cada pesquisador, seguida de verificação posterior entre eles. Identificaram-se também procedimentos nos quais os participantes integravam as etapas iniciais de análise de suas fotografias e/ou verificavam a análise realizada pelos pesquisadores expressando concordância ou solicitando correções.

\section{Discussão}

O uso de métodos visuais envolvendo fotografias tiradas pelos participantes parece ser recente no campo da saúde mental, uma vez que os maiores números de produção de artigos se concentram nos últimos três anos. Seu uso parece ser ainda mais recente no Brasil, consi- 
derando que foram encontrados apenas cinco artigos de autores brasileiros, dos quais apenas um atendeu aos critérios de inclusão deste trabalho (Carvalho et al., 2012). A maior prevalência de estudos foi identificada nos Estados Unidos, no Canadá, no Reino Unido, na Austrália e na Holanda.

O método fotográfico mais citado nos estudos foi o fotovoz. Entretanto, quando se verificou se os procedimentos utilizados nos estudos correspondiam às suas descrições e às denominações apontadas pelos autores principais de cada um dos métodos (Collier, 1957; Ziller \& Smith, 1977; Wang \& Buris, 1997; Sitvast \& Abma, 2011), identificou-se que, em muitos casos, os estudos contavam com utilizações inadequadas de suas denominações em relação aos procedimentos que realmente foram utilizados. O uso do termo fotovoz, por exemplo, foi identificado em procedimentos que não possuíam temas relacionados a uma comunidade, não contavam com discussões em grupo ou não estavam voltados ao objetivo de promover mudanças sociais. Essa inadequação é um ponto importante a ser destacado ao se considerar que ela pode ser responsável por confusões e incoerências na literatura. Além do questionamento emergente em relação à fidedignidade dos dados encontrados em revisões, pode-se questionar se o método mais utilizado foi realmente a fotovoz ou se, apesar de este ser o mais referido pelos autores, os procedimentos mais utilizados não corresponderiam de fato a outro método, como a fotoelicitação.

Apesar da confusão das denominações dos métodos e de apenas o fotovoz relacionar-se com bases teóricas da pesquisa-ação participativa, identificou-se que o uso de métodos fotográficos ou outros métodos visuais geralmente traz consigo propostas de quebra da relação tradicional pesquisador-participante, no qual o pesquisador é o responsável pelo delineamento da pesquisa, produção e disseminação do conhecimento, enquanto o participante é apenas o sujeito da pesquisa que Ihe fornece dados. A ideia de pedir ao participante para produzir fotos ou outros materiais visuais sobre algum tema busca aproximar pesquisadores e comunidade, colocando-os como igualmente contribuintes na produção do conhecimento (Cabassa et al., 2013) e dando voz aos participantes por meio dessa mudança nas relações de poder. Tais relações podem se configurar de diversas formas em uma pesquisa, seja no momento de definir seu tema e formato, falar sobre os materiais produzidos ou de analisá-los.

Quando se considera o uso de fotografias em uma pesquisa, deve-se levar em conta algumas questões relativas ao objetivo do trabalho, como: "Qual seria a relevância cultural e contextual de utilizarmos imagens para tratarmos determinado assunto?", "Qual é a conexão dos participantes com o mecanismo proposto?", "Qual é a relevância do uso de imagens e entrevistas para responder à pergunta de pesquisa?", Quais são os riscos e benefícios desse contexto específico?" e "Que embasamento teórico e metodológico justifica seu uso?".

Nesse sentido, houve muitas referências semelhantes a respeito dos propósitos que levaram os autores a optar pelo uso de métodos fotográficos em suas pesquisas, parecendo 
haver consenso quanto ao fato de a fotografia ser um instrumento para compartilhar e explorar experiências internas e auxiliar os profissionais na compreensão e no tratamento da experiência vivida pela população em questão.

Em relação aos principais efeitos relatados após o uso de métodos fotográficos, emergiram frequentemente relatos sobre: 1. o empoderamento dos participantes, com citações referentes à sensação de autonomia, valorização e motivação; 2. o processo de autorreflexão crítica sobre vida pessoal, contando com a reconstrução de significados para experiências e com uma maior consciência de si mesmo e seu processo de recuperação; 3. o efeito das fotografias como pontes entre experiências vividas e articulação verbal das experiências, contribuindo para os efeitos descritos pelos participantes e para o levantamento de informações relevantes a profissionais da saúde.

Exemplos práticos de tais efeitos são ilustrados por estudos empíricos como o de Quaglietti (2018), o qual possuía como objetivo examinar os benefícios do uso da fotografia como uma intervenção terapêutica em outros cuidados ambulatoriais de saúde mental. O estudo contou com uso da técnica fotovoz e foi realizado com 31 veteranos de guerra vivenciando processos de estresse pós-traumático, depressão e doenças mentais severas. Realizaram- se encontros em grupo uma vez por semana, por seis semanas. A cada encontro, os participantes recebiam a tarefa de fotografar e levar determinado número de fotos contando sua história de recuperação. Nos encontros seguintes, os participantes eram solicitados a falar sobre as fotografias de acordo com um roteiro de perguntas. As discussões foram gravadas e transcritas. Ao final do estudo, cada um dos participantes deveria ter produzido 25 fotos, das quais escolheriam seis para realização de uma exposição coletiva sobre suas histórias de recuperação. Ao início e ao fim de cada encontro, eles também respondiam a questionários Likert a fim de medir os efeitos da intervenção. Como resultados, Quaglietti (2018) relata que as fotografias ajudaram os participantes em seu processo de autoconhecimento, facilitaram o diálogo sobre experiências de recuperação que eram difíceis de ser compartilhadas e deram um senso de propósito à recuperação, permitindo que compreendessem como as ideias de recuperação poderiam ser alteradas à medida que os pensamentos e as emoções fossem explorados mais profundamente.

Diferentemente do consenso em relação aos propósitos e efeitos do uso de métodos fotográficos, a análise dos dados parece ser um aspecto ainda controverso quando considera a ampla variedade de metodologias e abordagens utilizadas. Essa grande diversidade pode ser atribuída ao desafio da análise dos dados subjetivos presentes nas imagens e nos dados das entrevistas. Como um retrato de tal desafio, pôde-se notar a pouquíssima quantidade de estudos que consideram as fotografias dados a serem analisados de forma independente.

Segundo Drew e Guillemin (2014), apesar de pesquisadores que trabalham com métodos visuais reconhecerem a natureza expressiva e cultural envolvida na construção de ima- 
gens, há uma grande discussão a respeito dos processos envolvidos em suas análises. Enquanto alguns pesquisadores problematizam a abordagem realista das imagens como "verdades" e promovem o papel essencial do espectador (público ou o pesquisador) na interpretação de seu significado, outros problematizam a transitoriedade e validade dessa interpretação, valorizando apenas a interpretação própria do criador da imagem a ser acessada por meio de entrevistas e grupos focais. Há ainda um terceiro grupo que busca equilibrar tais pontos de vista e reconhecer o valor da interpretação tanto dos criadores das imagens quanto de seu público no processo de análise de dados, como é o caso da metodologia de "análise de estrutura interpretativa". ${ }^{5}$

Além dessa questão, identificou-se também o agrupamento de indivíduos com características muito diferentes em uma mesma população (por exemplo, doenças mentais severas, incluindo tanto depressão como esquizofrenia). Apesar de os estudos descreverem tais populações em relação a informações demográficas como idade, gênero e transtornos mentais apresentados, presença ou ausência de tratamento psicológico e uso de medicações para saúde mental, tais dados geralmente não são levados em conta separadamente na etapa de análise de dados e descrição dos resultados. Isso pode gerar a desconsideração de dados importantes e relevantes a uma população específica. Quando se agrupam, por exemplo, pessoas com depressão severa e aquelas com esquizofrenia, as fotografias e discussões de cada um desses grupos poderiam abordar temas muito distintos, mas as particularidades de cada um dos grupos deixariam de ser notadas ao agrupá-los em um grande grupo de doenças mentais severas e descrever os resultados de maneira generalizada.

Outra questão importante a ser destacada é que alguns cuidados éticos associados ao uso de métodos fotográficos diferem em relação aos cuidados comumente tomados em pesquisas psicológicas, sendo necessário levar em conta, por exemplo, além do Termo de Consentimento Livre e Esclarecido, a autorização dos participantes em relação ao direito de divulgação e uso de imagens tiradas por eles, e, no caso de fotografarem terceiros, a autorização destes também seria necessária. Tais autorizações são formalizadas por Termos de Consentimento de Direito e Uso de Imagem e geralmente são entregues no mesmo dia em que as instruções sobre as fotos são dadas aos participantes, solicitando sua devolução no momento da discussão sobre as fotografias. Além das questões relacionadas aos direitos de imagem, é importante considerar também que a tarefa de fotografar, refletir e falar a respeito das imagens pode trazer à tona sentimentos difíceis para os participantes (Padgett et al., 2013), sendo recomendado oferecer a possibilidade de possuírem auxílio de serviços de atendimento psicológico durante a participação nos procedimentos.

5 Tradução livre de "interpretative framework analysis" (Drew \& Guillemin, 2014). 
Por fim, este artigo buscou identificar, mapear e apresentar as possibilidades de uso de fotografias tiradas por participantes em pesquisas no contexto da saúde mental. Secundariamente, buscou-se dar um pontapé inicial na consolidação das terminologias adequadas a cada um dos métodos citados, assim como se buscou tornar tal metodologia de pesquisa mais acessível ao conhecimento de pesquisadores e profissionais no contexto da saúde mental por meio da apresentação de suas características e formas de aplicação.

Como limitações deste estudo, é possível que ele não englobe todas as denominações de métodos fotográficos existentes. Algumas delas podem não constar nas strings desta revisão pela sua ausência nos resultados das pesquisas ou por variações em suas traduções.

Além disso, por conta da escassez de artigos nacionais relacionados ao assunto, os resultados e as discussões estão bastante restritos à literatura estrangeira. Sendo assim, seria interessante que mais estudos de revisão relacionados ao uso de fotografia tiradas por participantes em contextos mais amplos, como o da saúde, fossem realizados para verificar como tais questões se manifestam no contexto brasileiro. De semelhante forma, a produção de mais artigos empíricos e teóricos que utilizem os métodos fotográficos citados se faz necessária para a construção de uma literatura mais ampla e concisa em relação ao uso de tais metodologias no contexto da saúde mental. 


\section{Referências}

Braun, V., \& Clarke, V. (2006). Using thematic analysis in psychology. Qualitative Research in Psychology, 3(2), 77-101. http://dx.doi.org/10.1191/1478088706qpo630a

Carvalho, M. A. A. S., Ximenes, V. M., \& Bosi, M. L. M. (2012). Processos de fortalecimento em um Movimento Comunitário de Saúde Mental no Nordeste do Brasil: Novos espaços para a loucura. Aletheia, (37), 162-176. http://pepsic.bvsalud.org/scielo.php?script=sci_arttext\&pid=S1413$03942012000100012 \& \operatorname{lng}=p t \&$ tlng=pt

Cabassa, L. J., Parcesepe, A., Nicasio, A., Baxter, E., Tsemberis, S., \& Lewis-Fernández, R., (2013). Health and wellness photovoice project: Engaging consumers with serious mental illness in health care interventions. Qualitative Health Research, 23(5), 618-630. https://doi. org/10.1177/1049732312470872

Charmaz, K. (2006). Constructing Grounded Theory: A Practical Guide through Qualitative Analysis. SAGE.

Clements, K. (2012). Participatory action research and photovoice in a psychiatric nursing/clubhouse collaboration exploring recovery narrative. Journal of Psychiatric and Mental Health Nursing, 19(9), 785-791. https://doi.org/10.1111/j.1365-2850.2011.01853.x

Collier, J. (1957). Photography in anthropology: A report on two experiments. American Anthropologist, 59, 843-859. https://doi.org/10.1525/aa.1957.59.5.02a00100

Creighton, G., Oliffe, J. L., Butterwick, S., \& Saewyc, E. (2013). After the death of a friend: Young men's grief and masculine identities. Social Science \& Medicine, 84, 35-43. https://doi.org/10.1016/j. socscimed.2013.02.022

Drew, S. E., Duncan, R. E., \& Sawyer, S. M. (2010). Visual storytelling: A beneficial but challenging method for health research with young people. Qualitative Health Research, 20(12), 1677-1688. https://doi.org/10.1177/1049732310377455

Drew, S., \& Guillemin, M. (2014). From photographs to findings: Visual meaning-making and interpretive engagement in the analysis of participant-generated images. Visual Studies, 29(1), 54-67. https://doi.org/10.1080/1472586X.2014.862994

Erdner, A., Andersson, L., Magnusson, A., \& Lutzen, K. (2009). Varying views of life among people with long-term mental illness. Journal of Psychiatric \& Mental Health Nursing, 16, 54-60. http:// dx.doi.org/10.1111/j.1365-2850.2008.01329.x

Glaw, X., Inder, K., Kable, A., \& Hazelton, M. (2017). Visual methodologies in qualitative research: Autophotography and photo elicitation applied to mental health research. International Journal of Qualitative Methods, 6, 1-8. https://doi.org/10.1177/1609406917748215

Greco, V., Lambert, C. H., \& Park, M. (2016). Being visible: PhotoVoice as assessment for children in a school-based psychiatric setting. Scandinavian Journal of Occupational Therapy, 24(3), 222232. https://doi.org/10.1080/11038128.2016.1234642

Maniam, Y., Kumaran, P., Lee, Y. P., Koh, J., \& Subramaniam, M. (2016). The journey of young people in an early psychosis program involved in participatory photography. British Journal of Occupational Therapy, 79(6), 368-375. https://doi.org/10.1177/0308022615621567 
Oliffe, J. L., Creighton, G., Robertson, S., Broom, A., Jenkins, E. K., Ogrodniczuk, J. S., \& Ferlatte, O. (2017). Injury, Interiority, and Isolation in Men's Suicidality. American Journal of Men's Health, 11(4), 888-899. https://doi.org/10.1177/1557988316679576

Padgett, D. K., Smith, B. T., Derejko, K. S., Henwood, B. F., \& Tiderington, E. (2013). A picture is worth ...? Photo elicitation interviewing with formerly homeless adults. Qualitative health research, 23(11), 1435-1444. https://doi.org/10.1177/1049732313507752

Paton, J., Horsfall, D., \& Carrington, A. (2018). Sensitive inquiry in mental health: A tripartite approach. International Journal of Qualitative Methods, 17(1), 1-11. https://doi.org/10.1177/ 1609406918761422

Piat, M., Seida, K., Sabetti, J., \& Padgett, D. (2017). (Em)placing recovery: Sites of health and wellness for individuals with serious mental illness in supported housing. Health \& Place, 47, 71-79. https://doi.org/10.1016/j.healthplace.2017.07.006

Quaglietti, S. (2018) Using photography to explore recovery themes with veterans. Journal of Creativity in Mental Health, 13(2), 220-230. https://doi.org/10.1080/15401383.2018.1425174

Russinova, Z., Gidugu, V., Bloch, P., Restrepo-Toro, M., \& Rogers, E. S. (2018). Empowering individuals with psychiatric disabilities to work: Results of a randomized trial. Psychiatric Rehabilitation Journal, 41(3), 196-207. https://doi.org/10.1037/prj0000303

Smith, B. T., Padgett, D. K., Choy-Brown, M., \& Henwood, B. F. (2015). Rebuilding lives and identities: The role of place in recovery among persons with complex needs. Health \& Place, 33, 109-117. https://doi.org/10.1016/j.healthplace.2015.03.002

Sibeoni, J., Costa-Drolon, E., Poulmarc'h, L., Colin, S., Valentin, M., Pradère, J., \& Revah-Levy, A. (2017). Photo-elicitation with adolescents in qualitative research: An example of its use in exploring family interactions in adolescent psychiatry. Child and adolescent psychiatry and mental health, 11, 49. https://doi.org/10.1186/s13034-017-0186-z

Sitvast, J. E., Abma, T. A., \& Widdershoven, G. M. A. (2010). Facades of suffering: Clients' photo stories about mental illness. Archives of Psychiatric Nursing, 24(5), 349-361. https://doi.org/10.1016/j.apnu.2010.02.004

Sitvast, J. E., \& Abma, T. A. (2011) The photo-instrument as a health care intervention. Health Care Anal, 20, 177-195. https://doi.org/10.1007/s10728-011-0176-x

Strauss A., \& Corbin J. (1990) Basics of Qualitative Research: Grounded Theory Procedures and Techniques. Sage.

Teti, M., Cheak-Zamora, N., Lolli, B., \& Maurer-Batjer, A. (2016). Reframing autism: Young adults with autism share their strengths through photo-stories. Journal of Pediatric Nursing, 31(6), 619-629. https://doi.org/10.1016/j.pedn.2016.07.002

Tittoni, J. (2009). Psicologia e Fotografia: Experiências em intervenções Fotográficas. Editora Dom Quixote.

Vélez-Grau, C. (2018). Using Photovoice to examine adolescents' experiences receiving mental health services in the United States. Health Promotion International, 34(5), 912-920. https://doi. org/10.1093/heapro/dayo43 
Vilà, M., Pallisera, M., \& Fullana, J. (2016). Exploring the present and projecting the future: People with severe mental illness speaking for themselves. International Journal of Qualitative Studies in Education, 29(9), 1118-1130. https://doi.org/10.1080/09518398.2016.1201164

Wang, C. C. (1999). Photovoice: A participatory action research strategy applied to women's health. Journal of Women's Health, 8(2), 185-192. https://doi.org/10.1089/jwh.1999.8.185

Wang, C., \& Burris, M. (1997). Photovoice: Concept, methodology, and use for participatory needs assessment. Health Education and Behavior, 24, 369-387. https://doi.org/10.1177/ 109019819702400309

Wang, C., Morrel-Samuels, S., Hutchison, P. M., Bell, L., \& Pestronk, R. M. (2004). Flint Photovoice: Community building among youths, adults, and policymakers. American Journal of Public Health, 94(6), 911-913. https://doi.org/10.2105/ajph.94.6.911

Werremeyer, A. B., Aalgaard-Kelly, G., \& Skoy, E. (2016) Using Photovoice to explore patients' experiences with mental health medication: A pilot study. Mental Health Clinician, 6(3), 142-153. https://doi.org/10.9740/mhc.2016.05.142

Ziller, R. (1990). Photographing the self: Methods for observing personal orientations. Sage.

Ziller, R. C., \& Smith, D. E. (1977). A phenomenological utilization of photographs. Journal of Phenomenological Psychology, 7, 172-182. https://doi.org/10.1163/156916277X00042

EQUIPE EDITORIAL

Editora-chefe

Cristiane Silvestre de Paula

Editores associados

Alessandra Gotuzo Seabra

Ana Alexandra Caldas Osório

Luiz Renato Rodrigues Carreiro

Maria Cristina Triguero

Veloz Teixeira

\section{Editores de seção}

"Avaliação Psicológica"

Alexandre Serpa

André Luiz de Carvalho

Braule Pinto

Luiz Renato Rodrigues Carreiro

Vera Lúcia Esteves Mateus

"Psicologia e Educação"

Alessandra Gotuzo Seabra

Carlo Schmidt

Regina Basso Zanon

\section{"Psicologia Social e Saúde das PRODUÇÃo EDITORIAL \\ Populações" \\ Enzo Banti Bissoli \\ Coordenação editorial \\ Ana Claudia de Mauro}

Marina Xavier Carpena

"Psicologia Clínica"

Carolina Andrea Ziebold Jorquera

Julia Garcia Durand

Natalia Becker

"Desenvolvimento Humano"

Maria Cristina Triguero

Veloz Teixeira

Rosane Lowenthal

\section{Suporte técnico}

Maria Fernanda Liuti

Bento da Silva

Camila Fragoso Ribeiro
Estagiários editoriais

Júlia Lins Reis

Élcio Carvalho

Pietro Menezes

Preparação de originais

Carlos Villarruel

\section{Revisão}

Vera Ayres

\section{Diagramação}

Acqua Estúdio Gráfico 